\title{
Nanopatterning of colloidal nanocrystals emitters dispersed in a PMMA matrix by e-beam lithography
}

\author{
Luigi Martiradonna *, Tiziana Stomeo, Milena De Giorgi, \\ Roberto Cingolani, Massimo De Vittorio \\ National Nanotechnology Laboratory (NNL) of CNR-INFM, University of Lecce, Via per Arnesano, Lecce I-73100, Italy
}

Available online 20 February 2006

\begin{abstract}
We report on the fabrication of periodic nanostructures embedding semiconductor colloidal nanocrystals (NCs) by directly exposing a polymer/NCs blend to electron beam lithography (EBL). Our technological approach for the fabrication of NCs-based photonic devices relies on the dispersion of $\mathrm{CdSe} / \mathrm{ZnS}$ core/shell NCs into a layer of polymethilmethacrylate (PMMA) positive electron resist, which is patterned by means of an EBL process. The presence of NCs in the resist did not modify the peculiar behaviour of PMMA, which was selectively removed from the regions exposed to the electron beam. The morphology of the sample was assessed by scanning electron microscopy and atomic force microscopy measurements. The optical analysis of the samples after the dispersion of the NCs into the PMMA matrix and the exposure to the e-beam showed the successful localization of the colloidal NCs, whose emission properties were preserved.
\end{abstract}

(C) 2006 Elsevier B.V. All rights reserved.

Keywords: Colloidal nanocrystals; Electron beam lithography; Electroresist; Nanopositioning

\section{Introduction}

The interest for colloidal nanocrystals (NCs) in the area of photonics and optoelectronics is rapidly increasing. Nowadays low cost techniques $[1,2]$ with a high throughput based on direct chemical synthesis allow the fabrication of nanocrystals with different shapes [3] and with a tunability of the emission wavelength ranging from the ultraviolet [4] to the mid-infrared [5]. Several experiments on the gain properties of CdSe NCs showed that amplified spontaneous emission (ASE) and lasing can be achieved also at room temperature [6,7]. Moreover, the fluorescence efficiency and, in particular, the optical stability of the nanocrystals, can be greatly improved by modifying the particle surface in a core/shell configuration $[7,8]$. By virtue of these capping techniques, the optical properties remain unchanged also when the nanocrystals are dispersed into

\footnotetext{
* Corresponding author. Tel.: +39 832 298382; fax: +39 832298237.

E-mail address: luigi.martiradonna@unile.it (L. Martiradonna).
}

organic matrices. Merging the chemical stability of semiconductor NCs with the easy functionalization and lowcost processing of organic materials resulted to be advantageous in various applications [9]. Blends containing active fluorophores and nanocrystals have been used in order to study Förster energy transfer and charge transfer between the two emitters [10] and to obtain white emitting organic light emitting diodes (OLEDs) [11]. Blends with inert polymers such as polyisobutylmethacrylate or poly(methylmethachrylate) PMMA have been used in order to smoothen spin-coated films of nanocrystals [12] or in order to fabricate vertical microcavities having a well-defined resonance wavelength $[13,14]$. However, in all these samples the NCs active layer has been deposited by spin coating, without any control on the in-plane positioning.

The possibility of selectively localizing the NCs on a substrate is a very challenging task, because it can lead to the fabrication of two dimensional (2D) active photonic crystal (PC) devices or distributed feed-back (DFB) lasers without recurring to etching processes. 
In this work we have focused our attention on the precise localization of colloidal NCs through the nanopatterning of colloidal nanocrystals dispersed in a PMMA matrix. A blend of PMMA and CdSe/ZnS core/shell nanocrystals was directly exposed by means of an electron beam lithography process, in order to enable the fabrication of active PMMA-based optical devices embedding localized colloidal nanocrystals as the gain medium. Monodimensional periodic stripes and bidimensional periodic pillars have been patterned on a thin film of PMMA/NCs blend deposited on a silica substrate. Scanning electron microscopy (SEM) measurements were collected on the processed samples in order to check the surface morphology and to assess the effectiveness of the electron beam exposure on the blend film. The optical analysis, realized by means of photoluminescence (PL) maps recorded through a confocal microscope, showed that the optical properties of the colloidal nanoemitters were preserved also after the exposure to accelerated electrons.

\section{Experiment}

Nearly spherical, core/shell $\mathrm{CdSe} / \mathrm{ZnS}$ nanocrystals were prepared and diluted in chlorobenzene $\left(\mathrm{C}_{6} \mathrm{H}_{5} \mathrm{Cl}\right)$ by using standard methods $[15,16]$. The grown ensemble of nanoparticles showed an emission spectrum centered at $580 \mathrm{~nm}(\mathrm{FWHM}=40 \mathrm{~nm})$, with a residual presence of bigger aggregates emitting at $620 \mathrm{~nm}$. PMMA-950K positive resist diluted in chlorobenzene was chosen as the hosting polymer. The semiconductor clusters were mixed in solution phase in PMMA, with molar concentrations of $M_{\mathrm{NCs}}=5 \times 10^{-3} \mathrm{~mol} / 1$ and $M_{\mathrm{PMMA}}=1.5 \times 10^{-4} \mathrm{~mol} / \mathrm{l}$, respectively. The optical properties of the blend were assessed by photoluminescence (PL) measurements collected with a Cary Eclypse 5 Varian fluorimeter. Fig. 1 shows the emission spectra of the as-grown nanocrystals (solid line) and of the blend (dashed line): as expected,

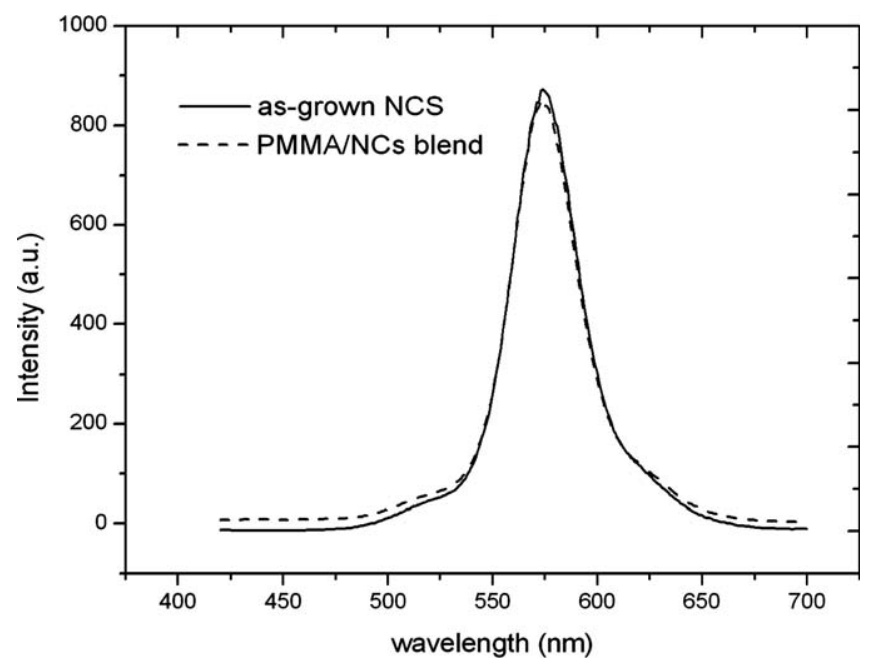

Fig. 1. PL analysis of the $\mathrm{CdSe} / \mathrm{ZnS}$ nanocrystals. Comparison between the emission of as-grown NCs, diluted in chorobenzene (solid line) and the emission of NCs dispersed in the PMMA matrix (dashed line). the two curves are perfectly superimposed, due to the optical transparency of PMMA in the visible spectral region.

Thick films $(250 \mathrm{~nm})$ of NCs/PMMA and pure PMMA were spin-coated on GaAs substrates. Both samples were annealed at $180{ }^{\circ} \mathrm{C}$ for $2 \mathrm{~min}$ in order to improve the film adhesion on the substrate; as verified in a previous work [14], the NCs/PMMA blend can bear these rapid high-temperature processing steps without undergoing to relevant optical degradation. Atomic force microscopy (AFM) was used to characterize the surface morphology of both samples. No increase of the surface roughness was detected on the sample containing NCs.

On the active NCs/PMMA film, two periodic test structures were defined by EBL with an acceleration voltage of $5 \mathrm{keV}$ and a beam current of $71 \mathrm{pA}$, carried out by a RAITH150 lithography system. The first periodic pattern consisted of a sequence of $2 \mu \mathrm{m}$ wide parallel stripes, with a period of $4 \mu \mathrm{m}$, repeated on a $50 \mu \mathrm{m} \times 50 \mu \mathrm{m}$ wide area. The second pattern consisted of a two dimensional triangular lattice of pillars having a diameter of $600 \mathrm{~nm}$ and a period of $2 \mu \mathrm{m}$. After the exposure, the sample was developed in a 1:3 mixture of methyl-isobutylketone/propanol (MIBK/IPA) at room temperature for $30 \mathrm{~s}$ and analyzed by scanning electron microscopy (SEM) in order to assess the surface morphology.

The optical properties of the blend after the EBL process were measured by room-temperature photoluminescence maps, collected by means of an OLYMPUS FluoView 1000 confocal laser scanning microscope, having a spatial resolution of $200 \mathrm{~nm}$. A laser diode emitting at $\lambda_{\mathrm{ex}}=405 \mathrm{~nm}$ with $40 \mu \mathrm{W}$ output power was used as the excitation source.

\section{Discussion}

Figs. 2(a) and (b) show the SEM top-view images collected on the processed patterns. The blend was completely removed from the regions exposed to the electron beam (bright regions), thus demonstrating that the presence of NCs in the resist did not modify the peculiar behaviour of PMMA as positive electron resist.

The PL maps of the samples in the spectral interval ranging from $560 \mathrm{~nm}$ to $600 \mathrm{~nm}$ were detected and reported in Figs. 3(a) and (b) for the periodic stripes and the two dimensional array of pillars, respectively. In this case, the bright patterns correspond to the unexposed PMMA/ NCs regions. With the same confocal microscope we also collected the emission spectrum of two different regions with a spectral resolution of $2 \mathrm{~nm}$. Fig. 3(c) shows the emission coming from the exposed areas (dotted line) and from the unexposed areas (dashed line). The absence of relevant PL signal among both the stripes and the pillars demonstrates that the $\mathrm{CdSe} / \mathrm{ZnS}$ nanocrystals dispersed in the blend were completely removed by MIBK developer, together with the exposed PMMA, without sticking to the substrate. This means that it is possible to have a perfect $\mathrm{ON}-\mathrm{OFF}$ control on the positioning of the nanoemit- 
(a)

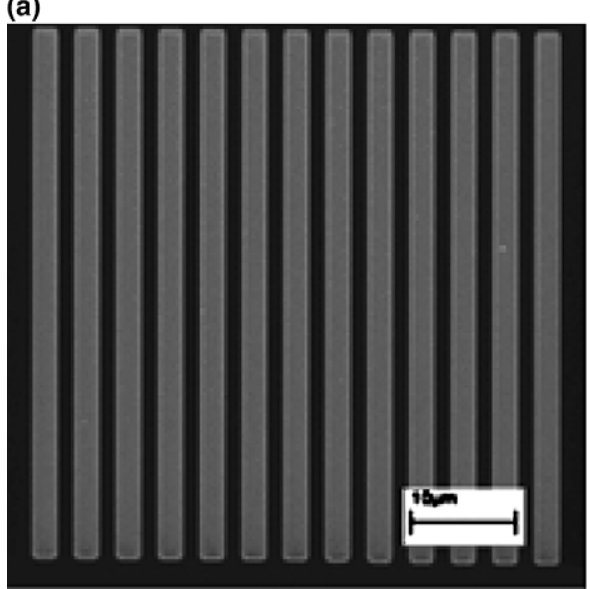

(b)

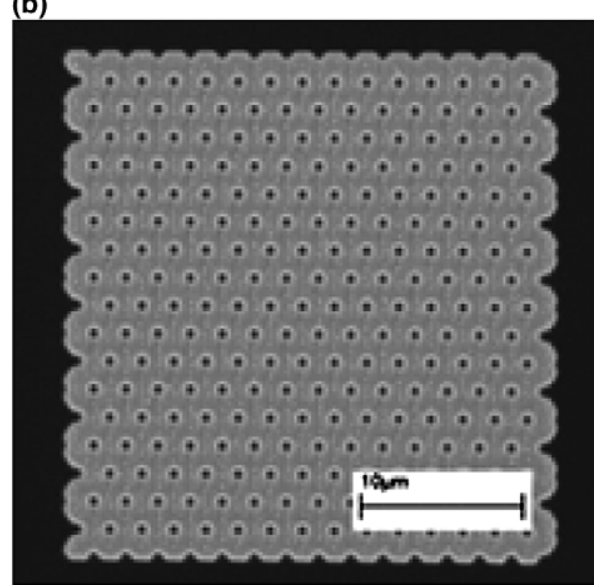

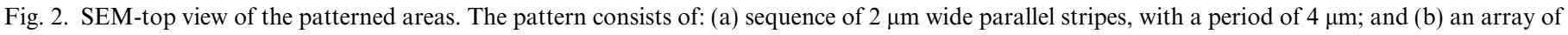
PMMA /NCs pillars (dark spots) with a diameter of $600 \mathrm{~nm}$ and a period of $2 \mu \mathrm{m}$.

(a)

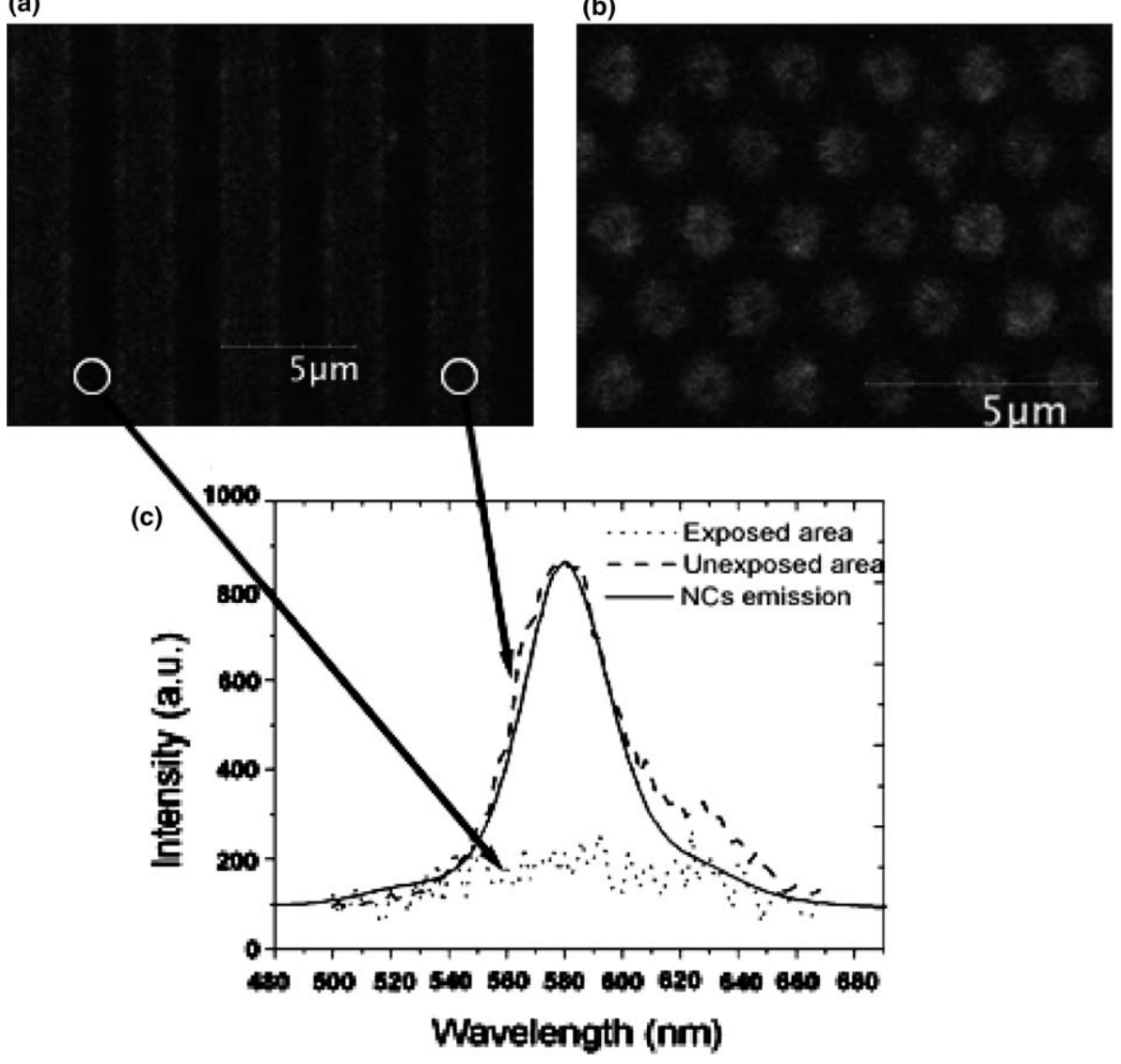

Fig. 3. (a, b) Photoluminescence(PL) maps of the patterned areas. The samples were excited by a 405 nm laser source. The PL map were detected at $580 \mathrm{~nm}$, corresponding to the emission peak of the semiconductor nanocrystals. (c) PL spectra coming from the exposed areas (dotted line), unexposed area (dashed line ) and of the as-grown NCs (solid line).

ters on the surface of the sample with the same resolution of traditional EBL processes.

We also compared the emission spectrum of NCs after the EBL process with the PL emission coming from the as-grown NCs (solid line in Fig. 3(c)). Disregarding the differences of intensity due to the different collecting systems used for the measurements, the emission spectrum remains clearly unaffected, confirming that nanocrystals do not undergo to relevant structural modifications or degradation of the optical properties.

In summary, we have successfully localized $\mathrm{CdSe} / \mathrm{ZnS}$ colloidal NCs dispersed in a PMMA matrix through e-beam lithography. The effectiveness of our process was demonstrated by room temperature photoluminescence (PL) maps 
recorded on the patterned samples, which show a complete removal of the active material from the regions exposed to electron beam without affecting the emission spectrum of NCs in the unexposed regions. Patterning the deposited active layer in order to selectively localize the NCs on a substrate is a very challenging task because it can lead to the fabrication of one dimensional (1D) or two dimensional (2D) active photonic crystal (PC) devices or distributed feed-back (DFB) lasers without recurring to etching processes. Moreover, by decreasing the concentration of nanocrystals in the blend and by isolating very small regions of the layer single $\mathrm{NC} /$ photon emitters can be enabled.

\section{Acknowledgments}

The authors gratefully thank Liberato Manna and Luigi Carbone for the chemical synthesis of $\mathrm{CdSe} / \mathrm{ZnS}$ nanocrystals and Gianmichele Epifani for his expert technical help.

\section{References}

[1] A. Kasuya, R. Sivamohan, Y.A. Barnakov, I.M. Nirasawa, V.R. Romanyuk, V. Kumar, S.V. Mamykin, K. Tohji, B. Jeyadevan, K. Shinoda, T. Kuda, O. Terasaki, Z. Liu, R.V. Belosludov, V. Sundararajan, Y. Kawazoe, Nat. Mater. 3 (2004) 99.
[2] A.P. Alivisatos, Science 271 (1996) 933.

[3] L. Manna, E.C. Scher, A.P. Alivisatos, J. Am. Chem. Soc. 122 (2000) 12700 .

[4] M.A. Hines, P. Guyot-Sionnest, J. Phys. Chem. B 102 (1998) 3655.

[5] J.M. Pietryga, R.D. Schaller, D. Werder, M.H. Stewart, V.I. Klimov, J.A. Hollingsworth, J. Am. Chem. Soc. 126 (2004) 11752.

[6] V.I. Klimov, A.A. Mikhailovsky, S. Xu, A. Malko, J.A. Hollingsworth, C.A. Leatgerdale, H.J. Eisler, M.G. Bawendi, Science 290 (2000) 314.

[7] Y. Chan, J.S. Steckel, P.T. Snee, J.M. Caruge, J.M. Hodgkiss, D.G. Nocera, M.G. Bawendi, Appl. Phys. Lett. 86 (2005) 073102.

[8] W.J. Parak, D. Gerion, T. Pellegrino, D. Zanchet, C. Micheel, S.C. Williams, R. Boudreau, M.A. Le Gros, C.A. Larabell, A.P. Alivisatos, Nanotechnology 14 (2003) R15.

[9] H. Du, G.Q. Xu, W.S. Chin, Chem. Mater. 14 (2002) 4473.

[10] M. Anni, L. Manna, R. Cingolani, D. Valerini, A. Creti, M. Lomascolo, Appl. Phys. Lett. 85 (2004) 4169.

[11] Y.Q. Li, A. Rizzo, M. Mazzeo, L. Carbone, L. Manna, R. Cingolani, G. Gigli, J. Appl. Phys. 97 (2005) 113501.

[12] J. Roither, W. Heiss, D.V. Talapin, N. Gaponik, A. Eychmuller, Appl. Phys. Lett. 84 (2004) 2223.

[13] C.E. Finlayson, D.S. Ginger, N.C. Greenham, Appl. Phys. Lett. 77 (2000) 2500.

[14] L. Martiradonna, L. Carbone, M. De Giorgi, T. Stomeo, M.T. Todaro, M. Anni, L. Manna, G. Gigli, R. Cingolani, M. De Vittorio, Proc. SPIE (2005) 168.

[15] P. Reiss, J. Bleuse, A. Pron, NanoLetters 7 (2002) 781

[16] B.O. Dabboussi, J. Rodriguez-Viejo, F.V. Mikulec, J.R. Heine, H. Mattoussi, R. Ober, K.F. Jensen, M.G. Bawendi, J. Phys. Chem. B 101 (1997) 9463. 